\title{
Strong ultra-regularity properties for positive elements in the twisted convolutions
}

\author{
Yuanyuan Chen ${ }^{1}$
}

Received: 25 February 2017 / Revised: 7 July 2017 / Accepted: 14 July 2017 /

Published online: 9 August 2017

(C) The Author(s) 2017. This article is an open access publication

\begin{abstract}
We show that positive elements with respect to the twisted convolutions, belonging to some ultra-test function space of certain order at origin, belong to the ultra-test function space of the same order everywhere. We apply the result to positive semi-definite Weyl operators.
\end{abstract}

Keywords Ultra-distributions · Twisted convolution · Hermite series expansions · Weyl quantization

\section{Introduction}

Several issues in operator theory can be studied by means of the twisted convolution. For example, composition and positivity questions can be carried over to related questions for the twisted convolution product by simple manipulations. We notice the simple structure of the twisted convolution, since it essentially consists of a convolution product, disturbed by a (symplectic) Fourier kernel. It is also common that boundedness, regularity and positivity conditions on operator kernels often correspond to convenient conditions on related elements in the twisted convolution. For example, operators with kernels in the Schwartz space $\mathscr{S}$, or in the Gelfand-Shilov spaces $\mathcal{S}_{S}$ or $\Sigma_{s}$ of Roumieu and Beurling types, respectively, can be transformed to twisted convolutions between elements in the same classes. (See Sect. 2 for notations.)

In [10] it is shown that various kinds of singularities for positive elements with respect to the twisted convolution are attained at the origin. Furthermore, it is proved

\footnotetext{
$\bowtie \quad$ Yuanyuan Chen

yuanyuan.chen@1nu.se

1 Department of Computer science, Physics and Mathematics, Linnæus University, Växjö, Sweden
} 
that regularity at origin for such elements impose global regularity and bounedness for these elements and their Fourier transforms.

More precisely, if $a \in \mathscr{D}^{\prime}$ is positive semi-definite with respect to the twisted convolution, then it is proved that the following is true:

(1) $a \in \mathscr{S}^{\prime}$ (cf. [10, Theorem 2.6]);

(2) if $\mathrm{WF}_{*}(a)$ is any wave-front set in Chapter VIII in [6] of $a$ and $(0, Y) \notin \mathrm{WF}_{*}(a)$, then $(X, Y) \notin \mathrm{WF}_{*}(a)$ and $(X, Y) \notin \mathrm{WF}_{*}\left(\mathscr{F}_{\sigma} a\right)$. Here $\mathscr{F}_{\sigma}$ is the symplectic Fourier transform (cf. [10, Theorem 4.14] and [11, Theorem 4.1]);

(3) if $a$ is continuous at origin, then $a$ and its Fourier transform $\widehat{a}$ are continuous everywhere and belong to $L^{2}$ (cf. [10, Theorem 3.13]);

(4) if $a \in C^{\infty}$ near origin, then $a \in \mathscr{S}$ (cf. [10, Theorem 3.13]);

(5) if $s \geq 0, a \in C^{\infty}$ near origin and

$$
\left|\partial^{\alpha} a(0)\right| \lesssim h^{|\alpha|} \alpha !^{s}
$$

for some $h>0$ (for every $h>0$ ), then $a \in \mathcal{S}_{S}\left(a \in \Sigma_{S}\right)$ (cf. [1, Theorem 4.1]).

We note that if (1.1) holds true with $s<1 / 2$ in (5), then $a$ is trivially equal to 0 , since the Gelfand-Shilov spaces $\mathcal{S}_{s}$ and $\Sigma_{s}$ are trivial for such choices of $s$.

In this paper we investigate related questions in background of Pilipović spaces, $\mathcal{S}_{s}$ and $\boldsymbol{\Sigma}_{s}$ of Roumieu and Beurling type respectively, a family of function spaces which agrees with corresponding Gelfand-Shilov spaces when these are non-trivial (cf. [8,9]). We introduce the so-called twisted Pilipović spaces $\mathcal{S}_{\sigma, s}$ and $\boldsymbol{\Sigma}_{\sigma, s}$ which are symplectic analogies of Pilipović spaces, and show that they are homeomorphic to $\mathcal{S}_{s}$ and $\boldsymbol{\Sigma}_{s}$, respectively. We also show that

$$
\mathcal{S}_{\sigma, s}=\mathcal{S}_{s}=\mathcal{S}_{S}
$$

when the right-hand side is non-trivial, and similarly for corresponding spaces of Beurling types.

We consider norm conditions of powers of a second order partial differential operator $H_{\sigma}$ and its conjugate. These operators are symplectic analogies to certain partial harmonic oscillators [7]. We show that $H_{\sigma}$ and $\bar{H}_{\sigma}$ commute and can be used to characterize $\mathcal{S}_{\sigma, s}$ and $\boldsymbol{\Sigma}_{\sigma, s}$ as

$$
a \in \mathcal{S}_{\sigma, s}\left(a \in \boldsymbol{\Sigma}_{\sigma, s}\right) \Leftrightarrow\left\|H_{\sigma}^{N_{1}} \bar{H}_{\sigma}^{N_{2}} a\right\|_{L^{\infty}} \lesssim h^{N_{1}+N_{2}}\left(N_{1} ! N_{2} !\right)^{2 s}
$$

for some $h>0$ (for every $h>0$ ). In Sect. 4 we show that if $a$ is positive semi-definite with respect to the twisted convolution, then the relaxed condition

$$
\left|H_{\sigma}^{N} \bar{H}_{\sigma}^{N} a(0)\right| \lesssim h^{N}(N !)^{4 s}
$$

of the right-hand of (1.2) is enough to ensure that $a$ should belong to $\mathcal{S}_{\sigma, s}$ or $\boldsymbol{\Sigma}_{\sigma, s}$. 


\section{Preliminaries}

In the first part we recall definitions of twisted convolution, the Weyl quantization and positivity in operator theory, and discuss basic properties. The verifications are in general omitted since they can be found in e. g. [10]. Thereafter we recall the definitions of Gelfand-Shilov and Pilipović spaces and discuss some properties.

\subsection{Operators and positivity}

Let $a$ and $b$ belong to $\mathscr{S}\left(\mathbf{R}^{2 d}\right)$, the set of Schwartz functions on $\mathbf{R}^{2 d}$. Then the twisted convolution of $a$ and $b$ is given by

$$
\left(a *_{\sigma} b\right)(X)=(2 / \pi)^{d / 2} \int_{\mathbf{R}^{2 d}} a(X-Y) b(Y) e^{2 i \sigma(X, Y)} d Y .
$$

Here $\sigma$ is the symplectic form on $\mathbf{R}^{d} \times \mathbf{R}^{d} \simeq \mathbf{R}^{2 d}$, given by

$$
\sigma(X, Y) \equiv\langle y, \xi\rangle-\langle x, \eta\rangle, \quad X=(x, \xi) \in \mathbf{R}^{2 d}, Y=(y, \eta) \in \mathbf{R}^{2 d}
$$

The definition of $*_{\sigma}$ extends in different ways. For example, the map $(a, b) \mapsto a *_{\sigma} b$ is continuous from $C_{0}^{\infty}\left(\mathbf{R}^{2 d}\right) \times C_{0}^{\infty}\left(\mathbf{R}^{2 d}\right)$ to $C_{0}^{\infty}\left(\mathbf{R}^{2 d}\right)$, and is uniquely extendable to a continuous map from $\mathscr{S}^{\prime}\left(\mathbf{R}^{2 d}\right) \times \mathscr{S}\left(\mathbf{R}^{2 d}\right)$ to $\mathscr{S}^{\prime}\left(\mathbf{R}^{2 d}\right)$, and from $\mathscr{D}^{\prime}\left(\mathbf{R}^{2 d}\right) \times C_{0}^{\infty}\left(\mathbf{R}^{2 d}\right)$ to $\mathscr{D}^{\prime}\left(\mathbf{R}^{2 d}\right)$.

There are strong links between the twisted convolution, and continuity and composition properties in operator theory. This also include analogous questions in the theory of pseudo-differential operators.

In fact, by straight-forward computations it follows that

$$
A\left(a *_{\sigma} b\right)=(A a) \circ(A b),
$$

where $A$ is the map on $\mathscr{S}\left(\mathbf{R}^{2 d}\right)$ defined by the formula

$$
(A a)(x, y)=(2 \pi)^{-d / 2} \int_{\mathbf{R}^{d}} a((y-x) / 2, \xi) e^{-i\langle x+y, \xi\rangle} d \xi
$$

(Here and in what follows we identify operators with their kernels.) We note that

$$
(A a)(x, y)=\left(\mathscr{F}^{-1}(a((y-x) / 2, \cdot))\right)(-(x+y))
$$

where $\mathscr{F}$ is the Fourier transform on $\mathscr{S}^{\prime}\left(\mathbf{R}^{d}\right)$ which takes the form

$$
\mathscr{F} f(\xi)=\widehat{f}(\xi) \equiv(2 \pi)^{-d / 2} \int_{\mathbf{R}^{d}} f(x) e^{-i\langle x, \xi\rangle} d x
$$


when $f \in \mathscr{S}\left(\mathbf{R}^{d}\right)$. Alternatively we may reformulate this identity as

$$
(A a)(x, y)=\left(\mathscr{F}_{2}^{-1} a\right)((y-x) / 2,-(x+y)),
$$

where $\mathscr{F}_{2} \Phi$ is the partial Fourier transform of $\Phi(x, y)$ with respect to the $y$-variable. Evidently, the mappings $\mathscr{F}_{2}$ and the pullback which takes $\Phi(x, y)$ into

$$
\Phi((y-x) / 2,-(x+y))
$$

are homeomorphisms on $\mathscr{S}\left(\mathbf{R}^{2 d}\right)$ and on $\mathscr{S}^{\prime}\left(\mathbf{R}^{2 d}\right)$, and unitary on $L^{2}\left(\mathbf{R}^{2 d}\right)$. Hence similar facts hold true for $A$.

From these mapping properties it follows that $A$ on $\mathscr{S}\left(\mathbf{R}^{2 d}\right)$ extends uniquely to a homeomorphism on $\mathscr{S}^{\prime}\left(\mathbf{R}^{2 d}\right)$, and if $a \in \mathscr{S}^{\prime}\left(\mathbf{R}^{2 d}\right)$, then $A a$ is a linear and continuous operator from $\mathscr{S}\left(\mathbf{R}^{d}\right)$ to $\mathscr{S}^{\prime}\left(\mathbf{R}^{d}\right)$. Furthermore, by the kernel theorem of Schwartz it follows that any linear and continuous operator from $\mathscr{S}\left(\mathbf{R}^{d}\right)$ to $\mathscr{S}^{\prime}\left(\mathbf{R}^{d}\right)$ is given by $A a$, for a uniquely determined $a \in \mathscr{S}^{\prime}\left(\mathbf{R}^{2 d}\right)$.

At this stage we also note that (2.1) remains true, if more generally, $a \in \mathscr{S}^{\prime}\left(\mathbf{R}^{2 d}\right)$ and $b \in \mathscr{S}\left(\mathbf{R}^{2 d}\right)$, which follows by straight-forward computations.

The operator $A$ can also in convenient ways be formulated in the framework of the Weyl calculus of pseudo-differential operators. More precisely, the Weyl quantization $\mathrm{Op}^{w}(a)$ of $a \in \mathscr{S}\left(\mathbf{R}^{2 d}\right)$ (the symbol) is the operator from $\mathscr{S}\left(\mathbf{R}^{d}\right)$ to $\mathscr{S}\left(\mathbf{R}^{d}\right)$ given by

$$
\mathrm{Op}^{w}(a) f(x)=(2 \pi)^{-d} \iint_{\mathbf{R}^{2 d}} a((x+y) / 2, \xi) f(y) e^{i\langle x-y, \xi\rangle} d y d \xi
$$

The definition of $\mathrm{Op}^{w}(a)$ extends in continuous and similar ways as for $A a$ to any $\mathscr{S}^{\prime}\left(\mathbf{R}^{2 d}\right)$, and then $\mathrm{Op}^{w}(a)$ is continuous from $\mathscr{S}\left(\mathbf{R}^{d}\right)$ to $\mathscr{S}^{\prime}\left(\mathbf{R}^{d}\right)$. This extension can also be performed by the relation

$$
\mathrm{Op}^{w}(a)=(2 \pi)^{-d / 2} A\left(\mathscr{F}_{\sigma} a\right)
$$

which follows by straight-forward computations. Here $\mathscr{F}_{\sigma}$ is the symplectic Fourier transform on $\mathscr{S}^{\prime}\left(\mathbf{R}^{2 d}\right)$, which takes the form

$$
\left(\mathscr{F}_{\sigma} a\right)(X) \equiv \pi^{-d} \int_{\mathbf{R}^{2 d}} a(Y) e^{2 i \sigma(X, Y)} d Y
$$

when $a \in \mathscr{S}\left(\mathbf{R}^{2 d}\right)$.

From these facts it follow that the Weyl product \#, defined by

$$
\mathrm{Op}^{w}(a \# b)=\mathrm{Op}^{w}(a) \circ \mathrm{Op}^{w}(b)
$$

is given by

$$
a \# b=(2 \pi)^{d / 2} a *_{\sigma}\left(\mathscr{F}_{\sigma} b\right)
$$

which again links the twisted convolution to compositions in operator theory. 
There are also strong links between positivity for the twisted convolution and positivity in operator theory. We recall that a continuous and linear operator $T$ from $\mathscr{S}\left(\mathbf{R}^{d}\right)$ to $\mathscr{S}^{\prime}\left(\mathbf{R}^{d}\right)$ (from $C_{0}^{\infty}\left(\mathbf{R}^{d}\right)$ to $\mathscr{D}^{\prime}\left(\mathbf{R}^{d}\right)$ ) is called positive semi-definite, whenever $(T f, f) \geq 0$ for every $f \in \mathscr{S}\left(\mathbf{R}^{d}\right)\left(f \in C_{0}^{\infty}\left(\mathbf{R}^{d}\right)\right)$, and then we write $T \geq 0$. Since $C_{0}^{\infty}\left(\mathbf{R}^{d}\right)$ is dense in $\mathscr{S}\left(\mathbf{R}^{d}\right)$, it follows that an operator from $\mathscr{S}\left(\mathbf{R}^{d}\right)$ to $\mathscr{S}^{\prime}\left(\mathbf{R}^{d}\right)$ is positive semi-definite, if it is positive semi-definite as an operator from $C_{0}^{\infty}\left(\mathbf{R}^{d}\right)$ to $\mathscr{D}^{\prime}\left(\mathbf{R}^{d}\right)$.

Positivity for the twisted convolution is defined in an analogous way. That is, an element $a \in \mathscr{S}^{\prime}\left(\mathbf{R}^{2 d}\right)\left(a \in \mathscr{D}^{\prime}\left(\mathbf{R}^{2 d}\right)\right)$ is positive semi-definite with respect to the twisted convolution, whenever $\left(a *_{\sigma} \varphi, \varphi\right) \geq 0$ for every $\varphi \in \mathscr{S}\left(\mathbf{R}^{2 d}\right)\left(\varphi \in C_{0}^{\infty}\left(\mathbf{R}^{2 d}\right)\right)$. As above it follows that $a \in \mathscr{S}^{\prime}\left(\mathbf{R}^{2 d}\right)$ is positive semi-definite with respect to $*_{\sigma}$, if it is positive semi-definite as an element in $\mathscr{D}^{\prime}\left(\mathbf{R}^{2 d}\right)$.

The following proposition explains the links between positivity in operator theory and positivity for the twisted convolution. Here $W_{f, g}$ is the Wigner distribution of $f \in \mathscr{S}^{\prime}\left(\mathbf{R}^{d}\right)$ and $g \in \mathscr{S}^{\prime}\left(\mathbf{R}^{d}\right)$, given by $W_{f, g} \equiv A^{-1}(\check{f} \otimes \bar{g})$. Here $\check{f}(x)=f(-x)$. If $f, g \in \mathscr{S}\left(\mathbf{R}^{d}\right)$, then $W_{f, g}$ takes the form

$$
W_{f, g}(x, \xi)=(2 \pi)^{-d / 2} \int_{\mathbf{R}^{d}} f(x-y / 2) \overline{g(x+y / 2)} e^{i\langle y, \xi\rangle} d y .
$$

Proposition 1 Let $a \in \mathscr{S}^{\prime}\left(\mathbf{R}^{2 d}\right)$. Then the following conditions are equivalent:

(1) a is positive semi-definite with respect to the twisted convolution;

(2) Aa is a positive semi-definite operator from $\mathscr{S}\left(\mathbf{R}^{d}\right)$ to $\mathscr{S}^{\prime}\left(\mathbf{R}^{d}\right)$;

(3) $\mathrm{Op}^{w}\left(\mathscr{F}_{\sigma}\right.$ a) is a positive semi-definite operator from $\mathscr{S}\left(\mathbf{R}^{d}\right)$ to $\mathscr{S}^{\prime}\left(\mathbf{R}^{d}\right)$;

(4) $\left(\mathscr{F}_{\sigma} a, W_{f, f}\right) \geq 0$ for every $f \in \mathscr{S}\left(\mathbf{R}^{d}\right)$.

\subsection{Gelfand-Shilov spaces}

Let $h, s \in \mathbf{R}_{+}$be fixed. Then $\mathcal{S}_{s, h}\left(\mathbf{R}^{d}\right)$ is the set of all $f \in C^{\infty}\left(\mathbf{R}^{d}\right)$ such that

$$
\|f\|_{\mathcal{S}_{s, h}} \equiv \sup \frac{\left|x^{\beta} \partial^{\alpha} f(x)\right|}{h^{|\alpha+\beta|}(\alpha ! \beta !)^{s}}
$$

is finite. Here the supremum is taken over all $\alpha, \beta \in \mathbf{N}^{d}$ and $x \in \mathbf{R}^{d}$.

The set $\mathcal{S}_{s, h}\left(\mathbf{R}^{d}\right)$ is a Banach space which increases with $h$ and $s$, and is contained in $\mathscr{S}\left(\mathbf{R}^{d}\right)$. If $s>1 / 2$, then $\mathcal{S}_{s, h}$ and $\cup_{h>0} \mathcal{S}_{1 / 2, h}$ are dense in $\mathscr{S}$. Hence, the dual $\left(\mathcal{S}_{s, h}\right)^{\prime}\left(\mathbf{R}^{d}\right)$ of $\mathcal{S}_{s, h}\left(\mathbf{R}^{d}\right)$ is a Banach space which contains $\mathscr{S}^{\prime}\left(\mathbf{R}^{d}\right)$.

The Gelfand-Shilov spaces $\mathcal{S}_{S}\left(\mathbf{R}^{d}\right)$ and $\Sigma_{S}\left(\mathbf{R}^{d}\right)$ are the inductive and projective limits respectively of $\mathcal{S}_{s, h}\left(\mathbf{R}^{d}\right)$ with respect to $h>0$. Consequently

$$
\mathcal{S}_{S}\left(\mathbf{R}^{d}\right)=\bigcup_{h>0} \mathcal{S}_{s, h}\left(\mathbf{R}^{d}\right) \quad \text { and } \quad \Sigma_{S}\left(\mathbf{R}^{d}\right)=\bigcap_{h>0} \mathcal{S}_{s, h}\left(\mathbf{R}^{d}\right),
$$

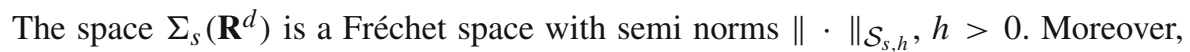
$\mathcal{S}_{S}\left(\mathbf{R}^{d}\right) \neq\{0\}$, if and only if $s \geq 1 / 2$, and $\Sigma_{S}\left(\mathbf{R}^{d}\right) \neq\{0\}$, if and only if $s>1 / 2$. 
If $\varepsilon>0$ and $s>0$, then

$$
\Sigma_{S}\left(\mathbf{R}^{d}\right) \subseteq \mathcal{S}_{S}\left(\mathbf{R}^{d}\right) \subseteq \Sigma_{S+\varepsilon}\left(\mathbf{R}^{d}\right)
$$

The Gelfand-Shilov distribution spaces $\mathcal{S}_{S}^{\prime}\left(\mathbf{R}^{d}\right)$ and $\Sigma_{s}^{\prime}\left(\mathbf{R}^{d}\right)$ are the projective and inductive limits respectively of $\mathcal{S}_{s, h}^{\prime}\left(\mathbf{R}^{d}\right)$. Hence

$$
\mathcal{S}_{S}^{\prime}\left(\mathbf{R}^{d}\right)=\bigcap_{h>0} \mathcal{S}_{s, h}^{\prime}\left(\mathbf{R}^{d}\right) \text { and } \Sigma_{s}^{\prime}\left(\mathbf{R}^{d}\right)=\bigcup_{h>0} \mathcal{S}_{s, h}^{\prime}\left(\mathbf{R}^{d}\right) .
$$

We note that $\mathcal{S}_{S}^{\prime}$ and $\Sigma_{s}^{\prime}$ are the duals of $\mathcal{S}_{s}$ and $\Sigma_{s}$, respectively, in view of [8,9].

The Gelfand-Shilov spaces and their duals are invariant under translations, dilations, (partial) Fourier transformations and under several other important transformations. In fact, by straight-forward computations it follows that the properties and results in Sect. 2.1 hold true with $\mathcal{S}_{s}$ and $\mathcal{S}_{S}^{\prime}$ in place of $\mathscr{S}$ and $\mathscr{S}^{\prime}$, respectively, when $s \geq 1 / 2$, or with $\Sigma_{s}$ and $\Sigma_{s}^{\prime}$ in place of $\mathscr{S}$ and $\mathscr{S}^{\prime}$, respectively, when $s>1 / 2$. We refer to [3] for more facts about Gelfand-Shilov functions and their distributions.

\subsection{The Pilipović spaces}

We start to consider spaces which are obtained by suitable estimates of Gelfand-Shilov or Gevrey type when using powers of the harmonic oscillator $H=|x|^{2}-\Delta, x \in \mathbf{R}^{d}$. In general we omit the arguments, since more thorough exposition is available in e.g. [13].

Let $s \geq 0$ and $h>0$. Then $\mathcal{S}_{h, s}\left(\mathbf{R}^{d}\right)$ is the Banach space which consists of all $f \in C^{\infty}\left(\mathbf{R}^{d}\right)$ such that

$$
\|f\| \mathcal{S}_{h, s} \equiv \sup _{N \geq 0} \frac{\left\|H^{N} f\right\|_{L^{\infty}}}{h^{N}(N !)^{2 s}}<\infty .
$$

If $h_{\alpha}$ is the Hermite function

$$
h_{\alpha}(x)=\pi^{-\frac{d}{4}}(-1)^{|\alpha|}\left(2^{|\alpha|} \alpha !\right)^{-\frac{1}{2}} e^{\frac{|x|^{2}}{2}}\left(\partial^{\alpha} e^{-|x|^{2}}\right)
$$

on $\mathbf{R}^{d}$ of order $\alpha$, then $H h_{\alpha}=(2|\alpha|+d) h_{\alpha}$. This implies that $\mathcal{S}_{h, s}\left(\mathbf{R}^{d}\right)$ contains all Hermite functions when $s>0$, and if $s=0$ and $\alpha \in \mathbf{N}^{d}$ satisfies $2|\alpha|+d \leq h$, then $h_{\alpha} \in \mathcal{S}_{h, s}\left(\mathbf{R}^{d}\right)$.

We let

$$
\boldsymbol{\Sigma}_{s}\left(\mathbf{R}^{d}\right) \equiv \bigcap_{h>0} \mathcal{S}_{h, s}\left(\mathbf{R}^{d}\right) \text { and } \mathcal{S}_{s}\left(\mathbf{R}^{d}\right) \equiv \bigcup_{h>0} \mathcal{S}_{h, s}\left(\mathbf{R}^{d}\right)
$$

and equip these spaces by projective and inductive limit topologies, respectively, of $\mathcal{S}_{h, s}\left(\mathbf{R}^{d}\right), h>0$. (Cf. [4,8,9,13].) 
The space $\boldsymbol{\Sigma}_{s}\left(\mathbf{R}^{d}\right)^{1}$ is called the Pilipović space (of Beurling type) of order $s \geq 0$ on $\mathbf{R}^{d}$. Similarly, $\mathcal{S}_{S}\left(\mathbf{R}^{d}\right)$ is called the Pilipović space (of Roumieu type) of order $s \geq 0$ on $\mathbf{R}^{d}$. Evidently, $\boldsymbol{\Sigma}_{0}\left(\mathbf{R}^{d}\right)$ is trivially equal to $\{0\}$, while

$$
h_{\alpha} \in \mathcal{S}_{s}\left(\mathbf{R}^{d}\right), \quad \text { when } s \geq 0 \quad \text { and } \quad h_{\alpha} \in \mathbf{\Sigma}_{s}\left(\mathbf{R}^{d}\right), \quad \text { when } s>0 \text {. }
$$

The dual spaces of $\mathcal{S}_{h, s}\left(\mathbf{R}^{d}\right), \boldsymbol{\Sigma}_{s}\left(\mathbf{R}^{d}\right)$ and $\mathcal{S}_{S}\left(\mathbf{R}^{d}\right)$ are denoted by $\mathcal{S}_{h, s}^{\prime}\left(\mathbf{R}^{d}\right), \boldsymbol{\Sigma}_{s}^{\prime}\left(\mathbf{R}^{d}\right)$ and $\mathcal{S}_{s}^{\prime}\left(\mathbf{R}^{d}\right)$, respectively. We have

$$
\Sigma_{s}^{\prime}\left(\mathbf{R}^{d}\right)=\bigcup_{h>0} \mathcal{S}_{h, s}^{\prime}\left(\mathbf{R}^{d}\right)
$$

when $s>0$ and

$$
\mathcal{S}_{S}^{\prime}\left(\mathbf{R}^{d}\right)=\bigcap_{h>0} \mathcal{S}_{h, s}^{\prime}\left(\mathbf{R}^{d}\right)
$$

when $s \geq 0$, with inductive respective projective limit topologies of $\mathcal{S}_{h, s}^{\prime}\left(\mathbf{R}^{d}\right), h>0$ (cf. [13]).

Let $s>0$ and $\varepsilon>0$. Then it follows from the definitions that

$$
\begin{aligned}
\mathcal{S}_{0}\left(\mathbf{R}^{d}\right) \subseteq \boldsymbol{\Sigma}_{s}\left(\mathbf{R}^{d}\right) \subseteq \mathcal{S}_{s}\left(\mathbf{R}^{d}\right) \subseteq \boldsymbol{\Sigma}_{s+\varepsilon}\left(\mathbf{R}^{d}\right) \subseteq \mathscr{S}\left(\mathbf{R}^{d}\right) \\
\subseteq \mathscr{S}^{\prime}\left(\mathbf{R}^{d}\right) \subseteq \boldsymbol{\Sigma}_{s+\varepsilon}^{\prime}\left(\mathbf{R}^{d}\right) \subseteq \mathcal{S}_{S}^{\prime}\left(\mathbf{R}^{d}\right) \subseteq \boldsymbol{\Sigma}_{S}^{\prime}\left(\mathbf{R}^{d}\right) \subseteq \mathcal{S}_{0}^{\prime}\left(\mathbf{R}^{d}\right)
\end{aligned}
$$

Furthermore, in [13] it is proved that $\mathcal{S}_{0}\left(\mathbf{R}^{d}\right)$ consists of all finite linear combinations of Hermite functions, while $\mathcal{S}_{0}^{\prime}\left(\mathbf{R}^{d}\right)$ consists of all formal series

$$
f=\sum_{\alpha \in \mathbf{N}^{d}} c_{\alpha} h_{\alpha}, \quad \text { where } c_{\alpha}=c_{\alpha}(f)=\left(f, h_{\alpha}\right)_{L^{2}}
$$

The next propositions show that Pilipović spaces can be characterized by estimates on the Hermite coefficients $c_{\alpha}$ in (2.6). The proofs can be found in [2,13] and therefore omitted. Here $H_{1} U$ and $H_{2} U$ are the partial harmonic oscillators given by

$$
H_{1} U(x, y)=\left(|x|^{2}-\Delta_{x}\right) U(x, y), \quad H_{2} U(x, y)=\left(|y|^{2}-\Delta_{y}\right) U(x, y) .
$$

Proposition 2 Let $s \geq 0(s>0)$ and $f \in \mathcal{S}_{0}^{\prime}\left(\mathbf{R}^{d}\right)$ be given by (2.6). Then the following conditions are equivalent:

(1) $f \in \mathcal{S}_{S}\left(\mathbf{R}^{d}\right)\left(f \in \boldsymbol{\Sigma}_{S}\left(\mathbf{R}^{d}\right)\right)$;

(2) $\left|c_{\alpha}(f)\right| \lesssim e^{-r|\alpha|^{\frac{1}{2 s}}}$ for some $r>0$ (for every $r>0$ ).

\footnotetext{
1 The boldface characters $\Sigma_{S}, \mathcal{S}_{S}$, etc. denote Pilipović spaces, and non-boldface characters $\Sigma_{S}$, $\mathcal{S}_{S}$, etc. denote analogous Gelfand-Shilov spaces.
} 
Proposition 3 Let $p, q \in(0, \infty], p_{0} \in[1, \infty], s \geq 0(s>0), U \in \mathcal{S}_{0}^{\prime}\left(\mathbf{R}^{2 d}\right)$, and $H_{1}$ and $H_{2}$ be given by (2.7). Then the following conditions are equivalent:

(1) $U \in \mathcal{S}_{S}\left(\mathbf{R}^{2 d}\right)\left(U \in \boldsymbol{\Sigma}_{S}\left(\mathbf{R}^{2 d}\right)\right)$;

(2) $\left\|H_{1}^{N_{1}} H_{2}^{N_{2}} U\right\|_{L^{p_{0}}} \lesssim h^{N_{1}+N_{2}}\left(N_{1} ! N_{2} \text { ! }\right)^{2 s}$ for some $h>0($ for every $h>0)$;

(3) $\left\|H_{1}^{N_{1}} H_{2}^{N_{2}} U\right\|_{M^{p, q}} \lesssim h^{N_{1}+N_{2}}\left(N_{1} ! N_{2} \text { !) }\right)^{2 s}$ for some $h>0($ for every $h>0)$.

Remark 1 Let $\mathcal{S}_{s}$ and $\Sigma_{s}$ be the Gelfand-Shilov spaces of order $s \geq 0$. Then it is proved in $[8,9]$ that

$$
\mathcal{S}_{s_{1}}=\mathcal{S}_{s_{1}}, \quad \Sigma_{s_{2}}=\Sigma_{s_{2}}, \quad s_{1} \geq \frac{1}{2}, s_{2}>\frac{1}{2},
$$

and that

$$
\Sigma_{s_{2}} \neq \Sigma_{s_{2}}, \quad \text { when } \quad s_{2}=\frac{1}{2}
$$

The latter relation was extend in [13] into

$$
\mathcal{S}_{s_{1}} \neq \mathcal{S}_{s_{1}}=\{0\}, \quad \Sigma_{s_{2}} \neq \Sigma_{s_{2}}=\{0\}, \quad s_{1}<\frac{1}{2}, 0<s_{2} \leq \frac{1}{2} .
$$

Remark 2 In [13] it is proved that $\mathcal{S}_{S_{1}}$ and $\boldsymbol{\Sigma}_{S_{2}}$ are not invariant under dilations when $s_{1}<1 / 2$ and $s_{2} \leq 1 / 2$.

Remark 3 Let the hypothesis in Proposition 3 be fulfilled, and let $\langle x\rangle=1+|x|$ when $x \in \mathbf{R}^{d}$. By letting $N_{1}=N_{2}=N$, (2) and (3) in Proposition 3 take the forms

(2)' $\left\|H_{1}^{N} H_{2}^{N} U\right\|_{L^{p_{0}}} \lesssim h^{N} N !^{4 s}$ for some $h>0$ (for every $h>0$ );

(3)' $\left\|H_{1}^{N} H_{2}^{N} U\right\|_{M^{p, q}} \lesssim h^{N} N !^{4 s}$ for some $h>0$ (for every $h>0$ ).

The same arguments as in [2,13] imply that these conditions are equivalent, Furthermore, let $\widetilde{\mathcal{S}}_{s}\left(\mathbf{R}^{2 d}\right)\left(\widetilde{\boldsymbol{\Sigma}}_{S}\left(\mathbf{R}^{2 d}\right)\right)$ be the set of all $U \in \mathcal{S}_{0}^{\prime}\left(\mathbf{R}^{2 d}\right)$ such that

$$
\left|c_{\alpha}(U)\right| \lesssim e^{-r\left(\left\langle\alpha_{1}\right\rangle\left\langle\alpha_{2}\right\rangle\right)^{\frac{1}{4 s}}}, \quad \alpha=\left(\alpha_{1}, \alpha_{2}\right),
$$

for some $r>0$ (for every $r>0$ ). Then it follows by similar arguments as in [2,13] that

$$
(2)^{\prime} \Leftrightarrow(3)^{\prime} \Leftrightarrow U \in \widetilde{\mathcal{S}}_{s}\left(\mathbf{R}^{2 d}\right)\left(U \in \widetilde{\mathbf{\Sigma}}_{S}\left(\mathbf{R}^{2 d}\right)\right) .
$$

For future references we note that $\mathcal{S}_{s} \subseteq \widetilde{\mathcal{S}}_{s} \subseteq \mathcal{S}_{2 s}$ with strict inclusions.

\section{Twisted Pilipović spaces and their properties}

In this section we introduce twisted Pilipovic spaces as the counter images of the operator $A$ on Pilipović spaces, and deduce some basic properties. We also consider their distribution spaces.

We begin with some definitions. 
Definition 1 The Hermite-Wong function of order

$$
\alpha=\left(\alpha_{1}, \alpha_{2}\right) \in \mathbf{N}^{d} \times \mathbf{N}^{d} \simeq \mathbf{N}^{2 d}
$$

on $\mathbf{R}^{2 d}$ is given by

$$
\varrho_{\alpha} \equiv A^{-1}\left(h_{\alpha_{1}} \otimes h_{\alpha_{2}}\right)=A^{-1}\left(h_{\alpha_{1}} \otimes \overline{h_{\alpha_{2}}}\right)=(-1)^{\left|\alpha_{1}\right|} W_{h_{\alpha_{1}}, h_{\alpha_{2}}} .
$$

The Hermite-Wong functions were studied in different ways by M. W. Wong in $[14,15]$. By the definition it follows that

$$
\varrho_{\alpha}(X)=(2 \pi)^{-d / 2} \int_{\mathbf{R}^{d}} h_{\alpha_{1}}\left(\frac{y}{2}-x\right) \overline{h_{\alpha_{2}}\left(\frac{y}{2}+x\right)} e^{i\langle y, \xi\rangle} d y,
$$

when $\alpha=\left(\alpha_{1}, \alpha_{2}\right) \in \mathbf{N}^{2 d}$ and $X=(x, \xi) \in \mathbf{R}^{2 d}$.

We observe that the Hermite-Wong functions are eigenfunctions to $\mathscr{F}_{\sigma}$. More precisely, we have

$$
\mathscr{F}_{\sigma} \varrho_{\alpha_{1}, \alpha_{2}}=(-1)^{\left|\alpha_{1}\right|} \varrho_{\alpha_{1}, \alpha_{2}},
$$

which follows from the fact that $\mathscr{F}_{\sigma}\left(W_{f, g}\right)=W_{\check{f}, g}$ (see e.g. [5]).

Definition 2 Let $s>0$.

(1) The set $\mathcal{S}_{\sigma, 0}^{\prime}\left(\mathbf{R}^{2 d}\right)$ consists of all formal expansions

$$
a=\sum_{\alpha} c_{\alpha} \varrho_{\alpha}
$$

where $\left\{c_{\alpha}\right\}_{\alpha \in \mathbf{N}^{2 d}} \subseteq \mathbf{C}$.

(2) The set $\mathcal{S}_{\sigma, 0}\left(\mathbf{R}^{2 d}\right)$ consists of all expansions in (3.1) such that $c_{\alpha}$ are non-zero for at most finite numbers of $\alpha$.

(3) The set $\mathcal{S}_{\sigma, s}\left(\mathbf{R}^{2 d}\right)\left(\boldsymbol{\Sigma}_{\sigma, s}\left(\mathbf{R}^{2 d}\right)\right)$ consists of all expansions in (3.1) such that

$$
\left|c_{\alpha}\right| \lesssim e^{-c|\alpha|^{\frac{1}{2 s}}}
$$

for some $c>0$ (for every $c>0$ ).

(4) The set $\mathcal{S}_{\sigma, s}^{\prime}\left(\mathbf{R}^{2 d}\right)\left(\boldsymbol{\Sigma}_{\sigma, s}^{\prime}\left(\mathbf{R}^{2 d}\right)\right)$ consists of all expansions in (3.1) such that

$$
\left|c_{\alpha}\right| \lesssim e^{c|\alpha|^{\frac{1}{2 s}}}
$$

for every $c>0$ (for some $c>0$ ).

The spaces in Definition 2 are equipped by topologies in similar way as for the Pilipović spaces in [13].

The set $\mathcal{S}_{\sigma, s}\left(\mathbf{R}^{2 d}\right)\left(\boldsymbol{\Sigma}_{\sigma, s}\left(\mathbf{R}^{2 d}\right)\right)$ is called the twisted Pilipovic space of Roumieu type (Beurling type) of order $s$. It follows that the sets $\mathcal{S}_{\sigma, s}^{\prime}\left(\mathbf{R}^{2 d}\right)$ and $\boldsymbol{\Sigma}_{\sigma, s}^{\prime}\left(\mathbf{R}^{2 d}\right)$ are 
corresponding distribution spaces, since similar facts hold true for Pilipović space [13].

We extend the definition of $A$ on $\mathscr{S}$ by letting

$$
A a=\sum_{\alpha} c_{\alpha} h_{\alpha}
$$

when $a \in \mathcal{S}_{\sigma, 0}^{\prime}\left(\mathbf{R}^{2 d}\right)$ is giving by (3.1). It follows that $A$ is a homeomorphism from $\mathcal{S}_{\sigma, s}\left(\mathbf{R}^{2 d}\right)$ to $\mathcal{S}_{s}\left(\mathbf{R}^{2 d}\right)$, from $\boldsymbol{\Sigma}_{\sigma, s}\left(\mathbf{R}^{2 d}\right)$ to $\boldsymbol{\Sigma}_{s}\left(\mathbf{R}^{2 d}\right)$, and similarly for their duals. Since it is clear that $A$ is a homeomorphism on any Fourier invariant Gelfand-Shilov spaces, we get

$$
\mathcal{S}_{\sigma, s}\left(\mathbf{R}^{2 d}\right)=\mathcal{S}_{S}\left(\mathbf{R}^{2 d}\right)=\mathcal{S}_{s}\left(\mathbf{R}^{2 d}\right), \quad \text { when } s \geq 1 / 2
$$

and

$$
\boldsymbol{\Sigma}_{\sigma, s}\left(\mathbf{R}^{2 d}\right)=\boldsymbol{\Sigma}_{s}\left(\mathbf{R}^{2 d}\right)=\Sigma_{s}\left(\mathbf{R}^{2 d}\right), \quad \text { when } s>1 / 2,
$$

and similarly for corresponding distribution spaces.

Remark 4 Let $a \in \mathcal{S}_{\sigma, 0}^{\prime}\left(\mathbf{R}^{2 d}\right)$ be as in (3.1). Since $A$ is a homeomorphism on $\mathscr{S}\left(\mathbf{R}^{2 d}\right)$ and on $\mathscr{S}^{\prime}\left(\mathbf{R}^{2 d}\right)$, it follows from [12] that a belongs to $\mathscr{S}\left(\mathbf{R}^{2 d}\right)$ if and only if $c_{\alpha} \lesssim$ $\langle\alpha\rangle^{-N}$ for every $N \geq 0$. In the same way, $a \in \mathscr{S}^{\prime}\left(\mathbf{R}^{2 d}\right)$ if and only if $c_{\alpha} \lesssim\langle\alpha\rangle^{N}$ for some $N \geq 0$.

Next we discuss the partial harmonic oscillators $H_{1}$ and $H_{2}$ in Proposition 3, and their counter images under the operator $A$. We let $H_{\sigma}$ be the operator on $\mathscr{S}\left(\mathbf{R}^{2 d}\right)$, given by

$$
H_{\sigma}=\left(|X|^{2}-\frac{1}{4} \Delta_{X}\right)+\left\langle\xi, D_{x}\right\rangle-\left\langle x, D_{\xi}\right\rangle, \quad X=(x, \xi) \in \mathbf{R}^{2 d},
$$

and we let $T_{\sigma}=H_{\sigma} \circ \bar{H}_{\sigma}$. Here we note that the conjugate $\bar{H}_{\sigma}$ of $H_{\sigma}$ is given by

$$
\bar{H}_{\sigma}=\left(|X|^{2}-\frac{1}{4} \Delta_{X}\right)-\left\langle\xi, D_{x}\right\rangle+\left\langle x, D_{\xi}\right\rangle .
$$

The following lemma explains some spectral properties of the considered operators.

Lemma 1 Let $s \geq 0$. Then the following is true:

(1) the Hermite-Wong functions $\varrho_{\alpha}$ are eigenfuctions to $H_{\sigma}, \bar{H}_{\sigma}$ and $T_{\sigma}$, and

$$
H_{\sigma} \varrho_{\alpha_{1}, \alpha_{2}}=\left(2\left|\alpha_{1}\right|+d\right) \varrho_{\alpha_{1}, \alpha_{2}}, \quad \bar{H}_{\sigma} \varrho_{\alpha_{1}, \alpha_{2}}=\left(2\left|\alpha_{2}\right|+d\right) \varrho_{\alpha_{1}, \alpha_{2}},
$$

and

$$
T_{\sigma} \varrho_{\alpha_{1}, \alpha_{2}}=\left(2\left|\alpha_{1}\right|+d\right)\left(2\left|\alpha_{2}\right|+d\right) \varrho_{\alpha_{1}, \alpha_{2}}
$$


(2) $H_{\sigma}$ and $\bar{H}_{\sigma}$ restrict to homeomorphisms on $\mathcal{S}_{\sigma, s}\left(\mathbf{R}^{2 d}\right)$ and on $\boldsymbol{\Sigma}_{\sigma, s}\left(\mathbf{R}^{2 d}\right)$;

(3) the definitions of $H_{\sigma}$ and $\bar{H}_{\sigma}$ extend uniquely to homeomorphisms on $\mathscr{S}^{\prime}\left(\mathbf{R}^{2 d}\right)$, $\mathcal{S}_{\sigma, s}^{\prime}\left(\mathbf{R}^{2 d}\right)$ and on $\boldsymbol{\Sigma}_{\sigma, s}^{\prime}\left(\mathbf{R}^{2 d}\right)$.

For the proof, we shall make use of the operators

$$
\begin{aligned}
Z_{1, j} & =\frac{1}{2} \partial_{z_{j}}+\bar{z}_{j}, & \widetilde{Z}_{1, j} & =\frac{1}{2} \partial_{\bar{z}_{j}}-z_{j}, \\
Z_{2, j} & =\frac{1}{2} \partial_{\bar{z}_{j}}+z_{j}, & \widetilde{Z}_{2, j} & =\frac{1}{2} \partial_{z_{j}}-\bar{z}_{j},
\end{aligned}
$$

where

$$
\begin{aligned}
z_{j} & =x_{j}+i \xi_{j}, & \bar{z}_{j} & =x_{j}-i \xi_{j}, \\
\partial_{z_{j}} & =\partial_{x_{j}}-i \partial_{\xi_{j}}, & \partial_{\bar{z}_{j}} & =\partial_{x_{j}}+i \partial_{\xi_{j}},
\end{aligned}
$$

(see [15, Section 22]). By similar arguments as in the proof of Theorem 22.1 in [15] we get

$$
\begin{aligned}
& Z_{1, j} \varrho_{\alpha_{1}, \alpha_{2}}=\left(2\left|\alpha_{2, j}\right|\right)^{1 / 2} \varrho_{\alpha_{1}, \alpha_{2}-e_{j}}, \\
& \widetilde{Z}_{1, j} \varrho_{\alpha_{1}, \alpha_{2}}=-\left(2\left|\alpha_{2, j}\right|+2\right)^{1 / 2} \varrho_{\alpha_{1}, \alpha_{2}+e_{j}}, \\
& Z_{2, j} \varrho_{\alpha_{1}, \alpha_{2}}=-\left(2\left|\alpha_{1, j}\right|\right)^{1 / 2} \varrho_{\alpha_{1}-e_{j}, \alpha_{2}}, \\
& \widetilde{Z}_{2, j} \varrho_{\alpha_{1}, \alpha_{2}}=\left(2\left|\alpha_{1, j}\right|+2\right)^{1 / 2} \varrho_{\alpha_{1}+e_{j}, \alpha_{2}},
\end{aligned}
$$

where $e_{1}, \ldots, e_{d}$ is the standard basis in $\mathbf{R}^{d}$, i. e., $e_{j}=\left(\delta_{1, j}, \ldots, \delta_{d, j}\right), j=1, \ldots, d$, and $\delta_{i, j}$ is the Kroniker's delta function.

In view of (3.3), the operators $Z_{1, j}$ and $Z_{2, j}$ can be considered as symplectic analogies of annihilation operators, and $\widetilde{Z}_{1, j}$ and $\widetilde{Z}_{2, j}$ as symplectic analogies of creation operators.

Proof First we prove (1). By straight-forward computations, we obtain

$$
H_{\sigma}=-\frac{1}{2}\left(\sum_{j=1}^{d} Z_{2, j} \widetilde{Z}_{2, j}+\widetilde{Z}_{2, j} Z_{2, j}\right)
$$

and

$$
\bar{H}_{\sigma}=-\frac{1}{2}\left(\sum_{j=1}^{d} Z_{1, j} \widetilde{Z}_{1, j}+\widetilde{Z}_{1, j} Z_{1, j}\right) .
$$

Hence, by (3.3) we get

$$
H_{\sigma} \varrho_{\alpha_{1}, \alpha_{2}}=\left(2\left|\alpha_{1}\right|+d\right) \varrho_{\alpha_{1}, \alpha_{2}},
$$

and

$$
\bar{H}_{\sigma} \varrho_{\alpha_{1}, \alpha_{2}}=\left(2\left|\alpha_{2}\right|+d\right) \varrho_{\alpha_{1}, \alpha_{2}},
$$

and (1) follows. 
By (3.2), it follows that $H_{\sigma}$ and $\bar{H}_{\sigma}$ restrict to homeomorphisms on $\mathcal{S}_{\sigma, s}\left(\mathbf{R}^{2 d}\right)$ and on $\boldsymbol{\Sigma}_{\sigma, s}\left(\mathbf{R}^{2 d}\right)$, which gives (2).

If $a \in \mathcal{S}_{\sigma, s}^{\prime}\left(\mathbf{R}^{2 d}\right)$ and $b \in \mathcal{S}_{\sigma, s}\left(\mathbf{R}^{2 d}\right)$. We now let $H_{\sigma} a$ be defined by

$$
\left(H_{\sigma} a, b\right)_{L^{2}}=\left(a, H_{\sigma} b\right)_{L^{2}}
$$

as usual, which extends the definitions of $H_{\sigma}$ and $\bar{H}_{\sigma}$ to $\mathcal{S}_{\sigma, s}^{\prime}\left(\mathbf{R}^{2 d}\right)$. The extensions of these operators to $\boldsymbol{\Sigma}_{\sigma, s}^{\prime}\left(\mathbf{R}^{2 d}\right)$ and $\mathscr{S}^{\prime}\left(\mathbf{R}^{2 d}\right)$ are performed in similar ways. By (3.2), it follows that these extensions are unique.

The next lemma shows important links between the latter operators and partial harmonic oscillators.

Lemma 2 Let $H_{1}$ and $H_{2}$ be as in Proposition 3, and let a $\in \mathcal{S}_{\sigma, s}\left(\mathbf{R}^{2 d}\right)$. Then $H_{\sigma}$ and $\bar{H}_{\sigma}$ are commuting to each other, and

$$
A\left(H_{\sigma}^{N_{1}} \bar{H}_{\sigma}^{N_{2}} a\right)=H_{1}^{N_{1}} H_{2}^{N_{2}}(A a)
$$

for every interger $N_{1}, N_{2} \geq 0$. In particular, if $\left\{f_{k}\right\}_{k=1}^{\infty}$ and $\left\{g_{k}\right\}_{k=1}^{\infty}$ are sequences in $l^{2}\left(\mathbf{N} ; L^{2}\left(\mathbf{R}^{d}\right)\right)$, and a is given by

$$
a=\sum_{k=0}^{\infty} A^{-1}\left(f_{k} \otimes \overline{g_{k}}\right)
$$

then

$$
A\left(T_{\sigma}^{N} a\right)=\sum_{k=0}^{\infty}\left(H^{N} f_{k}\right) \otimes\left(\overline{H^{N} g_{k}}\right),
$$

where the series converge in $\mathscr{S}^{\prime}\left(\mathbf{R}^{2 d}\right)$.

Proof The commutation between $H_{\sigma}$ and $\bar{H}_{\sigma}$ follows if we prove (3.4). We recall the operators

$$
\begin{aligned}
P_{j} & =\frac{1}{2 i} \partial_{\xi_{j}}-x_{j}, & \Pi_{j} & =\frac{1}{2 i} \partial_{x_{j}}+\xi_{j}, \\
T_{j} & =\frac{1}{2 i} \partial_{\xi_{j}}+x_{j}, & \Theta_{j} & =\frac{1}{2 i} \partial_{x_{j}}-\xi_{j},
\end{aligned}
$$

and the relations

$$
\begin{array}{ll}
A\left(P_{j}^{2} a\right)=x_{j}^{2} A a, & A\left(\Pi_{j}^{2} a\right)=-\partial_{x_{j}}^{2}(A a), \\
A\left(T_{j}^{2} a\right)=y_{j}^{2} A a, & A\left(\Theta_{j}^{2} a\right)=-\partial_{y_{j}}^{2}(A a),
\end{array}
$$

from [1, Theorem 4.1].

By straight-forward computations we get

$$
\left(x_{j}^{2}-\partial_{x_{j}}^{2}\right)(A a)=A\left(\left(P_{j}^{2}+\Pi_{j}^{2}\right) a\right)=A\left(H_{\sigma, j} a\right),
$$


where $H_{\sigma, j}=\left(X_{j}^{2}-\frac{1}{4} \Delta_{X_{j}}\right)+\xi_{j} D_{x_{j}}-x_{j} D_{\xi_{j}}$.

Summing up over all $j$ gives

$$
H_{1}(A a)=A\left(H_{\sigma} a\right)
$$

In the same way we get

$$
H_{2}(A a)=A\left(\bar{H}_{\sigma} a\right)
$$

and the result follows by induction.

From these mapping properties, Proposition 3 can now be carried over to the case of twisted Pilipović spaces as follows.

Proposition 4 Let $p, q \in(0, \infty]$ and $p_{0} \in[1, \infty]$ and let $s \geq 0(s>0)$. Then the following conditions are equivalent.

(1) $a \in \mathcal{S}_{\sigma, s}\left(\mathbf{R}^{2 d}\right)\left(a \in \mathbf{\Sigma}_{\sigma, s}\left(\mathbf{R}^{2 d}\right)\right)$;

(2) $\left\|H_{\sigma}^{N_{1}} \bar{H}_{\sigma}^{N_{2}} a\right\|_{L^{p_{0}}} \lesssim h^{N_{1}+N_{2}}\left(N_{1} ! N_{2} \text { !) }\right)^{2 s}$ for some $h>0($ for every $h>0)$;

(3) $\left\|H_{\sigma}^{N_{1}} \bar{H}_{\sigma}^{N_{2}} a\right\|_{M^{p, q}} \lesssim h^{N_{1}+N_{2}}\left(N_{1} ! N_{2} \text { !) }\right)^{2 s}$ for some $h>0($ for every $h>0)$.

Proof Let $U=A a$. Since $M^{p_{1}}\left(\mathbf{R}^{2 d}\right) \subseteq M^{p, q}\left(\mathbf{R}^{2 d}\right) \subseteq M^{p_{2}}\left(\mathbf{R}^{2 d}\right)$, when $p_{1}=$ $\min (p, q)$ and $p_{2}=\max (p, q)$, we may assume that $p=q$.

Since $A$ is a homeomorphism on $M^{p}\left(\mathbf{R}^{2 d}\right)$, we get

$$
\left\|H_{\sigma}^{N_{1}} \bar{H}_{\sigma}^{N_{2}} a\right\|_{M^{p}} \asymp\left\|A\left(H_{\sigma}^{N_{1}} \bar{H}_{\sigma}^{N_{2}} a\right)\right\|_{M^{p}}=\left\|H_{1}^{N_{1}} H_{2}^{N_{2}} U\right\|_{M^{p}},
$$

and the equivalence between (3) and Proposition 3 (3) follows. The equivalence between (1) and (3) now follows from Proposition 3 and the fact that $A$ is a homeomorphism from $\mathcal{S}_{\sigma, s}\left(\mathbf{R}^{2 d}\right)$ to $\mathcal{S}_{S}\left(\mathbf{R}^{2 d}\right)$.

Finally by the embeddings

$$
M^{1}\left(\mathbf{R}^{2 d}\right) \subseteq L^{p_{0}}\left(\mathbf{R}^{2 d}\right) \subseteq M^{\infty}\left(\mathbf{R}^{2 d}\right),
$$

the equivalence between (2) and (3) now follows.

Corollary 1 If $s \geq 0$ and $a \in \mathcal{S}_{\sigma, s}\left(\mathbf{R}^{2 d}\right)\left(a \in \boldsymbol{\Sigma}_{\sigma, s}\left(\mathbf{R}^{2 d}\right)\right)$, then

$$
\left\|T_{\sigma}^{N} a\right\|_{L^{\infty}} \lesssim h^{2 N}(N !)^{4 s}
$$

holds for some $h>0$ (for every $h>0)$.

Remark 3 and Lemma 2 show that (3.7) is necessary but not sufficient in order for $a \in \mathcal{S}_{\sigma, s}\left(\mathbf{R}^{2 d}\right)$ or $a \in \boldsymbol{\Sigma}_{\sigma, s}\left(\mathbf{R}^{2 d}\right)$. 


\section{Twisted Pilipović space property for positive elements with respect to the twisted convolution}

We study positive elements with respect to twisted convolution in $\mathscr{S}^{\prime}$, having twisted Pilipović space regularities near the origin. We show that such elements are in $\mathcal{S}_{\sigma, s}$ or in $\Sigma_{\sigma, s}$.

The following theorem shows that the condition of the form (3.7) at origin is sufficient that the converse of Corollary 1 holds when dealing with positive semidefinite elements with respect to the twisted convolution.

Theorem 1 Let $s \geq 0$ and $a \in \mathscr{S}^{\prime}\left(\mathbf{R}^{2 d}\right)$ be positive semi-definite with respect to the twisted convolution. If a is smooth near origin and

$$
\left(T_{\sigma}^{N} a\right)(0) \lesssim h^{N}(N !)^{4 s}
$$

holds for some $h>0$ (for every $h>0)$, then $a \in \mathcal{S}_{\sigma, s}\left(\mathbf{R}^{2 d}\right)\left(a \in \boldsymbol{\Sigma}_{\sigma, s}\left(\mathbf{R}^{2 d}\right)\right)$.

For the proof we recall that the trace of $A a$ is given by $(\pi / 2)^{d / 2} a(0)$ when $a \in$ $\mathscr{S}\left(\mathbf{R}^{2 d}\right)$.

Proof We have $a \in \mathscr{S}\left(\mathbf{R}^{2 d}\right)$ in view of Theorem 3.13 in [10]. We may write $a=$ $\sum_{k} A^{-1}\left(f_{k} \otimes \overline{f_{k}}\right)$ for some sequence $\left\{f_{k}\right\}_{k=0}^{\infty}$ with convergence in $\mathscr{S}\left(\mathbf{R}^{d}\right)$. By Lemma 2, we obtain

$$
A\left(T_{\sigma}^{N} a\right)=\sum_{k}\left(H^{N} f_{k} \otimes \overline{H^{N} f_{k}}\right) .
$$

Let $K=\sum_{k} f_{k} \otimes \overline{f_{k}}$ be the kernel of $A a$. Then

$$
\begin{aligned}
\left\|H_{1}^{N_{1}} H_{2}^{N_{2}} K\right\|_{L^{2}}^{2} & \leq\left\|H_{1}^{N_{1}} H_{2}^{N_{2}} K\right\|_{\operatorname{Tr}}^{2} \\
& \left.=\| \sum\left(H^{N_{1}} f_{k}\right) \otimes \overline{\left(H^{N_{2}} f_{k}\right.}\right) \|_{\operatorname{Tr}}^{2} \\
& \left.\leq\left(\sum \|\left(H^{N_{1}} f_{k}\right) \otimes \overline{\left(H^{N_{2}} f_{k}\right.}\right) \|_{\operatorname{Tr}}\right)^{2} \\
& =\left(\sum\left\|H^{N_{1}} f_{k}\right\|_{L^{2}}\left\|H^{N_{2}} f_{k}\right\|_{L^{2}}\right)^{2} \\
& \leq\left(\sum\left\|H^{N_{1}} f_{k}\right\|_{L^{2}}^{2}\right)\left(\sum\left\|H^{N_{2}} f_{k}\right\|_{L^{2}}^{2}\right) \\
& =\left\|A\left(T_{\sigma}^{N_{1}} a\right)\right\|_{\operatorname{Tr}}\left\|A\left(T_{\sigma}^{N_{2}} a\right)\right\|_{\operatorname{Tr}} \\
& \asymp\left(T_{\sigma}^{N_{1}} a\right)(0)\left(T_{\sigma}^{N_{2}} a\right)(0) \lesssim h^{N}\left(N_{1} ! N_{2} !\right)^{4 s} .
\end{aligned}
$$

Here the second inequality follows from the triangle inequality, the second equality from the fact that $\left.\left(H^{N_{1}} f_{k}\right) \otimes \overline{\left(H^{N_{2}} f_{k}\right.}\right)$ is the kernel of a rank one operator, the third inequality from Cauchy-Schwartz inequality and the last step from the fact that 
$A\left(T_{\sigma}^{N} a\right)$ is the kernel of a positive semi-definite operator, giving that the trace and the trace norm are the same. Thus

$$
\left\|H_{1}^{N_{1}} H_{2}^{N_{2}} K\right\|_{L^{2}} \lesssim h^{N}\left(N_{1} ! N_{2} !\right)^{2 s},
$$

for some $h>0$ (for every $h>0$ ), giving that $K \in \mathcal{S}_{S}\left(\mathbf{R}^{2 d}\right)\left(K \in \boldsymbol{\Sigma}_{s}\left(\mathbf{R}^{2 d}\right)\right)$ in view of Proposition 3. Hence $a \in \mathcal{S}_{\sigma, s}\left(\mathbf{R}^{2 d}\right)\left(a \in \boldsymbol{\Sigma}_{\sigma, s}\left(\mathbf{R}^{2 d}\right)\right)$.

Proposition 5 Let $s \geq 0$ be real, and let $a \in \mathscr{S}^{\prime}\left(\mathbf{R}^{2 d}\right)$ be such that $\mathrm{Op}^{w}(a) \geq 0$. If

$$
\left(T_{\sigma}^{N}\left(\mathscr{F}_{\sigma} a\right)\right)(0) \lesssim h^{N}(N !)^{4 s}
$$

holds for some $h>0$ (for every $h>0)$, then $a \in \mathcal{S}_{\sigma, s}\left(\mathbf{R}^{2 d}\right)\left(a \in \boldsymbol{\Sigma}_{\sigma, s}\left(\mathbf{R}^{2 d}\right)\right)$.

Acknowledgements I thank Joachim Toft for valuable suggestions and support.

Open Access This article is distributed under the terms of the Creative Commons Attribution 4.0 International License (http://creativecommons.org/licenses/by/4.0/), which permits unrestricted use, distribution, and reproduction in any medium, provided you give appropriate credit to the original author(s) and the source, provide a link to the Creative Commons license, and indicate if changes were made.

\section{References}

1. Chen, Y., Toft, J.: Boundeness of Gevrey and Gelfand-Shilov kernels of positive semi-definite operators. J. Pseudo Differ. Oper. Appl. 6, 153-185 (2015)

2. Chen, Y., Signahl, M., Toft, J.: Factorizations and singular value estimates of operators with GelfandShilov and Piliopović kernels. arXiv:1511.06257 (2016)

3. Gel'fand, I.M., Shilov, G.E.: Generalized Functions, vol. 2. Acdemic press, Boston (1968)

4. Gramchev, T., Pilipović, S.: L. Rodino Classes of degenerate elliptic operators in Gelfand-Shilov spaces. In: Rodino, L., Wong, M.W. (eds.) New Developments in Pseudo-differential Operators, Operator Theory: Advances and Applications, vol. 189, pp. 15-31. Birkhäuser Verlag, Basel (2009)

5. Folland, G.B.: Harmonic Analysis in Phase Space. Princeton University Press, Princeton (1989)

6. Hörmander, L.: The Analysis of Linear Partial Differential Operators, vol. 1. Springer, Berlin (1983)

7. Molahajloo, S., Wong, M.W., Rodino, L.: Metaplectic equivalce of the Hierarchical twisted Laplacian. Oper. Theory Adv. Appl. 231, 77-84 (2013)

8. Pilipović, S.: Generalization of Zemanian spaces of generalized functions which have orthonormal series expansions. SIAM J. Math. Anal. 17, 477-484 (1986)

9. Pilipović, S.: Tempered ultradistributions. Boll. U.M.I. 7, 235-251 (1988)

10. Toft, J.: Positivity properties in noncommutative convolution algebras with applications in pseudodifferential calculus. Bull. Sci. Math. 127, 101-132 (2003)

11. Toft, J.: Wave front set of positive operators and for positive elements in non-commutative convolution algebras. Studia Math. 179, 63-80 (2007)

12. Toft, J.: Multiplication properties in Gelfand-Shilov pseudo-differential calculus. In: Molahajlo, S., Pilipović, S., Toft, J., Wong, M.W. (eds.) Pseudo- Differential Operators, Generalized Functions and Asymptotics, Operator Theory: Advances and Applications 231, pp. 117-172. Birkhäuser, Basel (2013)

13. Toft, J.: Images of function and distribution spaces under the Bargmann transform. J. Pseudo-Differ. Oper. Appl. 8, 83-139 (2017)

14. Wong, M.W.: Weyl transforms and a degenerate elliptic partial differential equation. Proc. R. Soc. Lond. Ser. A Math. Phys. Eng. Sci. 461, 3863-3870 (2005)

15. Wong, M.W.: Weyl transforms. Springer, New York (1998) 